\title{
RINGS CHARACTERIZED BY CYCLIC MODULES
}

\author{
by DINH VAN HUYNH and PHAN DAN
}

(Received 22 October, 1987; revised 10 June, 1988)

1. Introduction. $A$ ring $R$ is called right $\mathrm{PCI}$ if every proper cyclic right $R$-module is injective, i.e. if $C$ is a cyclic right $R$-module then $C_{R} \cong R_{R}$ or $C_{R}$ is injective. By [2] and [3], if $R$ is a non-artinian right PCI ring then $R$ is a right hereditary right noetherian simple domain. Such a domain is called a right PCI domain. The existence of right PCI domains is guaranteed by an example given in [2]. As generalizations of right PCI rings, several classes of rings have been introduced and investigated, for example right CDPI rings, right CPOI rings (see [8], [6]). In Section 2 we define right PCS, right CPOS and right CPS rings and study the relationship between all these rings.

In Section 3 we consider rings each of whose cyclic right modules is a direct sum of an injective module and a semisimple module (briefly, CIS rings). By [4, Theorem 2.6], a ring $R$ is CIS if and only if $R$ is a generalized uniserial ring with $J^{2}=0$, where $J$ is the Jacobson radical of $R$. We shall prove that a ring $R$ is CIS if and only if every right $R$-module is a direct sum of a projective module and a semisimple module.

2. Definitions and results. Throughout, rings mean associative rings with identity and all modules are unitary. For a module $M$ over a ring $R$, we write $M_{R}$ to indicate that $M$ is a right $R$-module, the socle of $M$ is denoted by $\operatorname{Soc}(M)$. If $M=\operatorname{Soc}(M), M$ is called semisimple. Following Smith [8], a ring $R$ is called right CPOI (resp. right CDPI) if every cyclic right $R$-module is projective or injective (resp. a direct sum of a projective module and an injective module). Clearly there are implications:

right $\mathrm{PCI} \quad \Rightarrow \quad$ right $\mathrm{CPOI} \quad \Rightarrow \quad$ right $\mathrm{CDPI}$.

Now, a ring $R$ is called right CPS if every cyclic right $R$-module is a direct sum of a projective module and a semisimple module. Further, a ring $R$ is called right PCS if every proper cyclic right $R$-module is semisimple. Finally, a ring $R$ is called right CPOS if every cyclic right $R$-module is projective or semisimple. From the definitions we easily see the implications:

$$
\text { right PCS } \quad \Rightarrow \quad \text { right CPOS } \quad \Rightarrow \quad \text { right CPS. }
$$

THEOREM 1. Let $R$ be a ring with Jacobson radical $J$.

(I) The following statements hold:

(a) $R$ is a right CPOI ring if and only if $R$ is right CPOS with $J=0$;

(b) $R$ is right $C P O S$ with $J \neq 0$ if and only if $R_{R}=U \oplus S$, where $U_{R}$ is uniform having (composition) length 2 and $S_{R}$ is semisimple;

(c) $R$ is right $P C I$ if and only if $R$ is right $P C S$ with $J=0$;

(d) $R$ is right $P C S$ with $J \neq 0$ if and only if $J$ is the maximal and minimal right ideal of $R$.

Glasgow Math. J. 31 (1989) 251-256. 
(II) If $R$ is right CPS then the following conditions are equivalent:

(i) $R$ is right $C D P I$;

(ii) $J_{R}$ is projective;

(iii) $R$ is right non-singular.

For the proof of Theorem 1 we need the following lemma.

Lemma 2. Let $R$ be a right CPS ring with Jacobson radical J. Then:

(a) $R_{R}$ is a direct sum of noetherian uniform modules;

(b) $J_{R}$ is semisimple.

Proof. By [1, Theorem 3.1], every right CPS ring is right noetherian. Hence $R$ has the following decomposition

$$
R_{R}=e_{1} R \oplus \ldots \oplus e_{n} R,
$$

where $\left\{e_{i}\right\}_{i=1}^{n}$ is a set of orthogonal idempotents of $R$ and each $e_{i} R$ is indecomposable. We shall show that each $e_{i} R$ is uniform.

We set $e=e_{i}$ for a fixed $i$ and consider the right $R$-module $e R$. Let $M$ be a non-zero submodule of $e R$. By assumption there are submodules $M_{1}, M_{2}$ of $e R$ containing $M$ such that

$$
e R / M=M_{1} / M \oplus M_{2} / M,
$$

where $M_{1} / M$ is projective and $M_{2} / M$ is semisimple. It follows that $e R / M_{2}$ is projective. Hence $e R=M_{2} \oplus C$ for some submodule $C$ of $e R$. Since $e R$ is indecomposable, $C$ has to be zero, i.e. $e R / M$ is semi-simple. Now let $H$ be a submodule of $e R$ with $M \cap H=0$. Then there exists a submodule $B$ of $e R$ containing $M$ such that

$$
e R / M=B / M \oplus(M+H) / M \text {. }
$$

From this it follows that $e R=B \oplus H$. Hence $H=0$, showing that $e R$ is uniform, i.e. (a) holds.

For (b), let $J\left(e_{i} R\right)$ be the Jacobson radical of $e_{i} R$. Then

$$
J=J\left(e_{1} R\right) \oplus \ldots \oplus J\left(e_{n} R\right) .
$$

By (a), for each non-zero submodule $M$ of $e_{i} R, e_{i} R / M$ is semisimple. Therefore $M$ is an intersection of finitely many maximal submodules of $e_{i} R$. It follows that $M \supseteq J\left(e_{i} R\right)$. This shows that $J\left(e_{i} R\right)$ is simple if $J\left(e_{i} R\right)$ is non-zero. Thus $J_{R}$ is semisimple.

Proof of (I) of Theorem 1. (a) Let $R$ be a right CPOI ring. By [6, Theorem] or by [8, Theorem 2.12], $R=A \oplus B$, where $A$ is a semiprime artinian ring and $B$ is a right CPI domain. Then $B$ is right noetherian and for each non-zero right ideal $B_{1}$ of $B, B / B_{1}$ is $B$-injective and each of its submodules is injective too. Therefore $B / B_{1}$ is injective and semisimple. From this we can easily see that $R$ is right CPOS with $J=0$.

Conversely, let $R$ be right CPOS with $J=0$. Then, in particular, $R$ is right CPS. By Lemma 2,

$$
R_{R}=e_{1} R \oplus \ldots \oplus e_{n} R
$$


where $\left\{e_{i}\right\}_{i=1}^{n}$ is a set of orthogonal idempotents of $R$ and each $e_{i} R$ is uniform. If $R$ is right artinian $R$ is semisimple, in particular $R$ is right CPOI. Suppose now that $R$ is not right artinian. Then there is an $e_{i} R, e_{1} R$ say, which is a non-artinian right $R$-module. Therefore $e_{1} R$ contains a non-zero submodule $M$ with $M \neq e_{1} R$. Since $e_{1} R$ is uniform, for such a submodule $M$ of $e_{1} R, e_{1} R / M$ is semisimple by hypothesis. Hence $\operatorname{Soc}\left(e_{1} R\right)=0$. Since

$$
R / M \cong e_{1} R / M \oplus e_{2} R \oplus \ldots \oplus e_{n} R
$$

with non-projective $e_{1} R / M, R / M$ has to be semisimple. Hence

$$
A:=\operatorname{Soc}\left(R_{R}\right)=e_{2} R \oplus \ldots \oplus e_{n} R .
$$

Put $B=e_{1} R$; we have $R_{R}=A \oplus B$ with $\operatorname{Soc}\left(B_{R}\right)=0$. Clearly $B A=0$. Furthermore, since $(A B)^{2}=A(B A) B=0$, it follows that $A B \subseteq J=0$, proving that $B$ is also an ideal of $R$. This shows that $R$ is a direct sum of a semiprime artinian $\operatorname{ring} A$ and a right CPS domain $B$. Now, for each non-zero right ideal $H$ of $B, B / H$ is a semisimple right $B$-module. By [8], it follows that $B$ is a right $V$-ring, i.e. each simple right $B$-module is injective. Hence $B / H$ is an injective right $B$-module, proving that $B$ is a right CPI domain. By [6, Theorem] or by [8, Theorem 2.12], $R$ is right CPOI.

(b) Let $R$ be a right CPOS ring with $J \neq 0$. As above, $R_{R}$ has a decomposition (1). Since $J \neq 0$, there is an $e_{i} R, e_{1} R$ say, such that $e_{1} R$ is not simple. Let $M$ be a proper non-zero submodule of $e_{1} R$. Then as we have seen above, $e_{1} R / M$ is semisimple and non-projective. Therefore $S=e_{2} R \oplus \ldots \oplus e_{n} R$ is also semisimple. We have $J \subseteq e_{1} R$. Hence by Lemma 2 and by the uniformity of $e_{1} R, J_{R}$ is simple. In particular $R$ is right artinian; so $\operatorname{End}_{R}\left(e_{1} R\right)$ is a local ring; it follows that $e_{1} R$ has a unique maximal submodule. Hence $J$ is the maximal and minimal submodule of $e_{1} R$, or in other words, $e_{1} R$ has composition length 2. Put $U=e_{1} R$ and we have the decomposition of $R$ in (b).

Conversely let $R$ be a ring with a decomposition as in (b). Then the Jacobson radical $J$ of $R$ is the minimal submodule of $U_{R}$. Let $A$ be a non-zero right ideal of $R$. It is enough to show that either $A_{R}$ is a direct summand of $R_{R}$ or $R / A_{R}$ is semisimple. Suppose that $A_{R}$ is not a direct summand of $R_{R}$. Then $A \cap J \neq 0$. It follows that $J \subseteq A$; therefore $R / A_{R}$ is semisimple.

Since every right PCI (resp. right CPS) ring is a right CPOI (resp. right CPOS) ring, one can easily prove (c) and (d) by using (a) and (b), respectively.

Proof of (II) of Theorem 1. (i) $\Rightarrow$ (iii) is clear.

(iii) $\Rightarrow$ (ii). By Lemma $2, J_{R}$ is semisimple. Let $M$ be any minimal submodule of $J_{R}$. Then for some non-zero $m \in M, M=m R$. Hence $M_{R} \cong R / r(m)$, where $r(m)=\{x \in R: m x=0\}$. By (iii), $r(m)$ is not essential in $R_{R}$. It follows that $R_{R}=r(m) \oplus H$ for some right ideal $H$ of $R$ which is $R$-isomorphic to $M_{R}$. Hence $M_{R}$ is projective, proving that $J_{R}$ is projective.

(ii) $\Rightarrow$ (i). Assume (ii). By Lemma 2, $J_{R} \subseteq \operatorname{Soc}\left(R_{R}\right)$. Then $J^{2}=0$ and $\operatorname{Soc}\left(R_{R}\right)=$ $J_{R} \oplus H$, where $H_{R}$ is a direct sum of finitely many minimal right ideals of $R$ (because a right CPS ring is right noetherian having finite uniform dimension). Thus we can easily check that $H_{R}$ is generated by an idempotent of $R$. Hence $\operatorname{Soc}\left(R_{R}\right)$ is projective. To end 
the proof it is enough to show that any non-projective simple right $R$-module is injective. Let $S$ be such a module and $U$ a right ideal of $R$ with a homomorphism $\varphi$ of $U_{R}$ to $S_{R}$. We show that $\varphi$ can be extended to an $R$-homomorphism of $R$ to $S$. Without loss of generality, we can assume that $U_{R}$ is essential in $R_{R}$. If $\varphi(U)=0$, the assertion is trivial. Suppose that $\varphi(U) \neq 0$. We have $S \cong U / \operatorname{ker} \varphi$. If $\operatorname{ker} \varphi$ is not essential in $U_{R}$ then $U_{R}=\operatorname{ker} \varphi \oplus T$. Hence $T \subseteq \operatorname{Soc}\left(R_{R}\right)$; so $T_{R}$ is projective, a contradiction to the non-projectivity of $S_{R}\left(\cong T_{R}\right)$. Hence $\operatorname{ker} \varphi$ has to be essential in $U_{R}$. It follows that $\operatorname{ker} \varphi$ is an essential right ideal of $R$. Since $R$ is right CPS, it follows that $R / \operatorname{ker} \varphi$ is semisimple. Hence there is a submodule $L$ of $R_{R}$ containing $\operatorname{ker} \varphi$ such that

$$
R_{R} / \operatorname{ker} \varphi=U / \operatorname{ker} \varphi \oplus L / \operatorname{ker} \varphi .
$$

Therefore $R=L+U$, $\operatorname{ker} \varphi=L \cap U, R / L \cong U / \operatorname{ker} \varphi \cong S$. Combining these facts, we get that $\varphi$ is extended to a homomorphism in $\operatorname{Hom}_{R}\left(R_{R}, S_{R}\right)$, proving the injectivity of $S_{R}$.

The proof of Theorem 1 is complete.

Examples. Let $\mathbb{R}$ and $\mathbb{C}$ be the fields of real and complex numbers, respectively. Then the matrix ring

$$
R=\left[\begin{array}{ll}
\mathbb{R} & \mathbb{C} \\
0 & \mathbb{C}
\end{array}\right]
$$

has a decomposition $R_{R}=U \oplus S$, where $U=\left[\begin{array}{ll}R & \mathbb{C} \\ 0 & 0\end{array}\right], S=\left[\begin{array}{ll}0 & 0 \\ 0 & \mathbb{C}\end{array}\right]$. Clearly $U_{R}$ is uniform, having composition length 2 , and $S_{R}$ is simple. Then, by Theorem 1 (I), (b), $R$ is a right CPOS ring. Moreover, $R$ has the following properties:

(1) $R$ is not a direct sum of a semiprime artinian ring and a right PCS domain;

(2) $R$ is not a right PCS ring;

(3) $R$ is not a right CPOI ring;

(4) $R$ is a right CPS ring which is not right CDPI;

(5) the ring theoretic direct sum $R \oplus R$ is a right CPS ring which is not right CPOS.

The above assertions can be proved easily by using Theorem 1 .

Denote by $\mathbb{Z}$ the ring of integers. Then $\mathbb{Z} / 4 \mathbb{Z}$ is a PCS ring which is not PCI.

We do not know an example of a right noetherian right CDPI ring which is not right CPS. We also do not know whether any right CDPI ring is right noetherian.

3. Characterizations of CIS rings. A module $M$ is called uniserial if the set of submodules of $M$ is linearly ordered. A ring $R$ is said to be right generalized uniserial if it is right artinian and $R_{R}$ is a direct sum of uniserial modules. A generalized uniserial ring is a ring which is right and left uniserial.

THEOREM 3. For a ring $R$ with Jacobson radical $J$ the following conditions are equivalent:

(a) every cyclic right $R$-module is a direct sum of an injective module and a semisimple module; 
(b) every right $R$-module is a direct sum of an injective module and a semisimple module;

(c) every right $R$-module is a direct sum of a projective module and a semisimple module;

(d) $R$ is a generalized uniserial ring with $J^{2}=0$.

Proof. (a) $\Leftrightarrow$ (b) $\Leftrightarrow$ (d) are proved in [4, Theorem 2.6].

(d) $\Rightarrow$ (c). Assume (d). Let $M$ be a right $R$-module. By [5, Theorem 25.4.2], $M=\oplus_{i} M_{i}$, where each $M_{i}$ is a finitely generated uniserial module. By [5, Theorem 18.23], for each $M_{i}$, there exists a primitive idempotent $e_{i}$ and a submodule $F$ of $e_{i} R$ such that $M_{i} \cong e_{i} R / F$. Since, by [4, Theorem 2.6], the length of $e_{i} R$ is at most $2, M_{i}$ is projective or simple. Hence $M$ is a direct sum of a projective module and a semisimple module.

(c) $\Rightarrow$ (d). Assume (c). By a well-known theorem of Kaplansky, every projective module is a direct sum of countably generated modules. Hence (c) says that every right $R$-module is a direct sum of countably generated modules. Hence by [5, Theorem 20.23], $R$ is right artinian. Since $R$ is right CPS, by Lemma $2, J_{R}$ is semisimple and hence $J^{2}=0$. Also, by Lemma $2, R_{R}=e_{1} R \oplus \ldots \oplus e_{n} R$, where $\left\{e_{i}\right\}_{i=1}^{n}$ is a set of orthogonal idempotents of $R$ and each $e_{i} R$ is uniform. Suppose that, for a fixed $i$, the length of $e_{i} R$ is greater than 1 and let $M$ be the minimal submodule of $e_{i} R$. By (c), $e_{i} R / M$ is semisimple, i.e. $M$ is an intersection of maximal submodules of $e_{i} R$. But since $R$ is right artinian, $e_{i} R$ has a unique maximal submodule. It follows that $M$ is a maximal submodule of $e_{i} R$. This shows that the length of $e_{i} R$ is 2 . Further, denote by $E\left(e_{i} R\right)$ the injective hull of $e_{i} R$. By (c), $E\left(e_{i} R\right)$ has to be projective because $e_{i} R$ is uniform and not simple. By [5, Theorem 20.15], $E\left(e_{i} R\right)$ is isomorphic to some $e_{j} R$ with $1 \leq j \leq n$. Hence $E\left(e_{i} R\right)$ has length 2 . It follows that $E\left(e_{i} R\right)=e_{i} R$, i.e. $e_{i} R$ is injective. Now (d) follows from [4, Theorem 2.6].

The proof of Theorem 3 is complete.

We note that the statements of Theorem 3 are left-right symmetric. For short we call a ring in Theorem 3 a CIS ring. One can prove that a ring $R$ with nilpotent Jacobson radical $J$ is generalized uniserial if and only if $R / J^{2}$ is a CIS ring.

Since a right PCI domain is not CIS, Theorem 3 is not true if the condition (c) is only required for cyclic right $\mathrm{R}$-modules.

Acknowledgement. The authors wish to express their gratitude to Patrick F. Smith for his many helpful comments and suggestions. The first author gratefully acknowledges the support of the Alexander von Humboldt-Stiftung for his research.

\section{REFERENCES}

1. A. W. Chatters, A characterisation of right Noetherian rings, Quart. J. Math. Oxford Ser. (2) 33 (1982), 65-69.

2. J. H. Cozzens and C. Faith, Simple Noetherian rings (Cambridge University Press, 1975). $11-14$.

3. R. F. Damiano, A right PCI ring is right Noetherian, Proc. Amer. Math. Soc. 77 (1979), 
4. Dinh van Huynh, Nguyen V. Dung and Patrick F. Smith, On rings characterized by their right ideals or cyclic modules, Proc. Edinburgh Math. Soc., to appear.

5. C. Faith, Algebra II: Ring theory (Springer, 1976).

6. S. C. Goel, S. K. Jain and S. Singh, Rings whose cyclic modules are injective or projective, Proc. Amer. Math. Soc. 53 (1975), 16-18.

7. G. O. Michler and $O$. E. Villiamayor, On rings whose simple modules are injective, $J$. Algebra 25 (1973), 185-201. 93-111.

8. P. F. Smith, Rings characterized by their cyclic modules, Canad. J. Math. 31 (1979),

INSTITUTE OF Mathematics

P.O. Box 631 Bo Hô

HANOI, VIETNAM 